UNIVERSITÀ DEGLI STUDI DI BERGAMO

DIPARTIMENTO DI INGEGNERIA DELL'INFORMAZIONE

E METODI MATEMATICI

QUADERNI DEL DIPARTIMENTO

Department of Information Technology and Mathematical Methods

Working Paper

Series "Mathematics and Statistics"

n. $8 / \mathrm{MS}-2010$

Influence of bicuspid valve geometry on ascending

aortic fluid-dynamics: a parametric study

by

C. Vergara, F. Viscardi, L. Antiga, G.B. Luciani 
COMITATO DI REDAZIONE ${ }^{\S}$

Series Information Technology (IT): Stefano Paraboschi

Series Mathematics and Statistics (MS): Luca Brandolini, Ilia Negri

\footnotetext{
$\S$ L'accesso alle Series è approvato dal Comitato di Redazione. I Working Papers della Collana dei Quaderni del Dipartimento di Ingegneria dell'Informazione e Metodi Matematici costituiscono un servizio atto a fornire la tempestiva divulgazione dei risultati dell'attività di ricerca, siano essi in forma provvisoria o definitiva.
} 


\title{
Influence of bicuspid valve geometry on ascending aortic fluid-dynamics: a parametric study
}

\author{
C. Vergara* F. Viscardi ${ }^{\dagger}$ L. Antiga $\ddagger$ G.B. Luciani ${ }^{\S}$
}

\begin{abstract}
Bicuspid aortic valve (BAV) predisposes to aortic aneurysms with a high prevalence. A first hypothesis of this phenomenon is related to fibrillin deficiency (genetic hypothesis). In this paper we focus on a complementary, haemodynamic hypothesis stating that is the peculiar fluid-dynamics of the blood in the ascending aorta in BAV configurations which leads to those conditions that facilitate the aneurysms' formation. To corroborate that hypothesis, we perform a parametric study based on numerical simulations of ascending aorta hemodynamics in different configurations of orifice area and valve orientation. We investigate the resulting WSS distributions and degrees of asymmetry of the blood jet, and we introduce surrogate indices which may find direct application in clinical environments.
\end{abstract}

Keywords: Bicuspid aortic valve, ascending aorta, computational models, wall shear stress, aortic aneurysms.

\section{Introduction}

Bicuspid aortic valve (BAV) is the most common congenital heart disease $(0.5-2 \%)$ [12]. It predisposes to aortic valve pathology and aortic aneurysms at different levels with a high prevalence (33\%) [32]. Even in patients with normally functioning aortic BAV, echocardiographic finding of aortic dilatation is common (50\%) [22]. The pathogenesis of congenital aortic valve disease is complex, with a phenotypic continuum of aortic valve configurations, from unicuspid to various forms of bicuspid aortic valve with equal or unequal cusps and with or without raphe. Classification systems for BAV are thus disparate (echographic, surgical, pathoanathomical), and failing to be comprehensive [24, 26, 29]. However, two common configurations can be recognized: antero-posterior (AP) BAV when there is an ideal fusion of the two coronary cusps, and latero-lateral (LL) BAV, when there is an ideal fusion of the right and noncoronary cusps [27]. These phenotypes are known to hold pathogenetic [10,11] and embryogenetic [13] differences. Pathogenesis of aortic dilatation in presence of BAV is controversial. Some authors underline the role of fibrillin deficiency, (due to down-regulation, or excessive degradation) and of eNOS, claiming a genetic hypothesis [18]. Aneurysmatic aortic wall in BAV patients shows peculiar features with respect to tricuspid aneurysms, such as impaired aortic wall elasticity, and lower elastin loss and elastic fibers

*Department of Information Technology and Mathematical Methods, University of Bergamo, Viale Marconi 5, 24044, Italy. Tel: +39 035 2052314. Fax: +39 035562779. E-mail:christian.vergara@unibg.it. Corresponding author.

${ }^{\dagger}$ Divisions of Cardiac Surgery, University of Verona, Italy

¥Biomedical Engineering Department, Mario Negri Institute, Bergamo, Italy

$\S$ Divisions of Cardiac Surgery, University of Verona, Italy 
fragmentation but more focal smooth muscle cell loss [6]. An alternative hemodynamic hypothesis states that flow patterns through an even non-stenotic bicuspid orifice may cause altered wall shear stress (WSS) and promote aneurysm formation. To support the latter theory, echocardiographic studies found maximum shear stress at ascending aortic wall convexity [3], while magnetic resonance showed abnormal aortic flow patterns [16], abnormal circumferential and positional WSS [33], and eccentric aortic jet [23] in $\mathrm{BAV}$ patients. Also, a correlation among eccentric jet, aortic diameters and matrix metalloprotheinasis-2 expression may suggest that hemodynamic factors (rather than genetic) contribute to MMP-2 expression, leading to ascending aortic aneurysm formation [23].

In a previous fluid-dynamics simulation study we confirmed these findings, with a quantification of aortic WSS in BAV patients [31]. However, that study was concentrated on only two valve configurations, taken as representative for the potentially infinite BAV phenotypes, and limited to qualitative considerations. Possibly the chosen orifice area of the aortic bicuspid valve and its orientation with respect to aortic geometry may have had an influence on the final conclusions.

In this paper, we perform a parametric study by varying the area and orientation of BAV, and investigate how bicuspid valve geometry may influence the localization and magnitude of WSS using quantitative indices. In addition, we provide quantitative insight on the asymmetry of the blood flow jet exiting from BAV valves, which in principle can be considered as a macroscopic, measurable surrogate of the impaired WSS distribution in the ascending aorta. Such evaluations are based on indices which have been designed here to be readily applicable as part of routine radiological evaluations.

\section{Material and methods}

\subsection{Reconstruction of geometries from MRI data}

A surface model of the aortic root, ascending aorta, aortic arch, and thoracic aorta of a subject affected by BAV are obtained from spin-echo MRI images [31] using a level-set segmentation technique as provided by the Vascular Modeling Toolkit (see [1]). In particular, the technique leads to the generation of a surface representing the lumen boundary located at the points of steepest intensity changes. An analytical model of a bicuspid valve orifice is mathematically defined on a two-dimensional plane by the intersection of two circle functions of different radii (see Figure 1). This function is sampled on the surface representing the

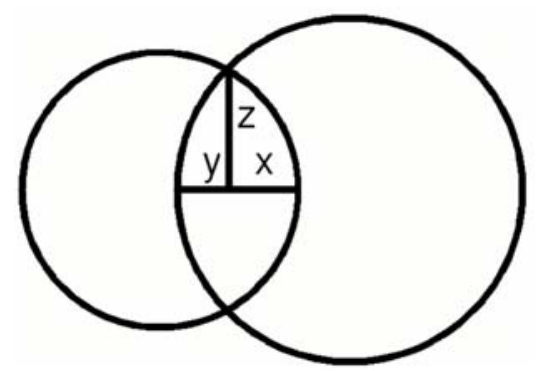

Figure 1: Intersection of two circle functions of different radii in order to determine the different valve configurations. Tricuspid valve corresponds to $x=y=z=9.8 \mathrm{~mm}$.

aortic root inlet and is used to open an orifice resembling typical bicuspid valves with given parameters 
AV area, AV orientation, position of the valve inside aorta.

In particular, we consider four AP configurations, depicted in Figure 2, namely a tricuspid configuration (TRI) modeled as a circle of area equal to $3.0 \mathrm{~cm}^{2}$, and three bicuspid configurations (two non-stenotic, B1 and B2, and one stenotic, B3), modeled as the union of two equal arches of circle, with area of $1.9 \mathrm{~cm}^{2}, 1.5 \mathrm{~cm}^{2}$ and $1.2 \mathrm{~cm}^{2}$, respectively. Moreover, in order to perform a sensitivity analysis
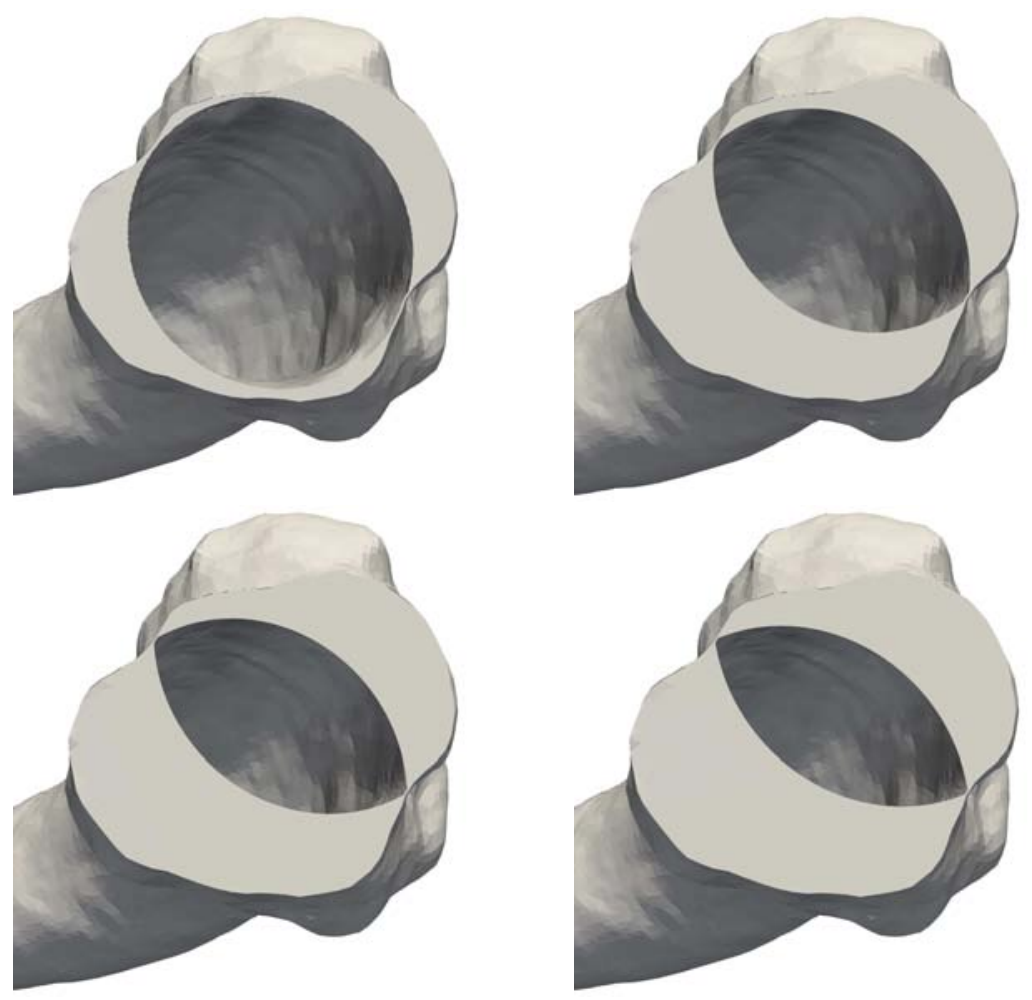

Figure 2: The four considered configurations: tricuspid valve TRI (left, up), bicuspid valve with area $=1.9 \mathrm{~cm}^{2}$ B1 (right, up), bicuspid valve with area $=1.5 \mathrm{~cm}^{2} \mathrm{~B} 2$ (left, bottom) and bicuspid valve with area $=1.2 \mathrm{~cm}^{2} \mathrm{~B} 3$ (right, bottom).

of the fluid-dynamics quantities with respect to the orientation of the valve, we consider six different orientations of the B2 configuration. In particular, we rotate the valve in steps of $30^{\circ}$ from its original position (B2-h12, see Figure 3). In what follows, we refer to configurations B2-h12, B2-h1 and B2-h5 as antero-posterior configurations, whilst to B2-h2, B2-h3 and B2-h4 as latero-lateral configurations.

The surface models are successively turned into volumetric meshes of linear tetrahedra in order for computational fluid-dynamics simulations to be carried out, using the meshing capabilities of the Vascular Modeling Toolkit. In all the meshes, we have about $1.3 \cdot 10^{6}$ tetrahedra. This size is reached after successive mesh refinements, with the aim of obtaining a mesh-independent numerical solution. An example of the relation between patient MRI and resulting model is reported in Figure 4, left. 

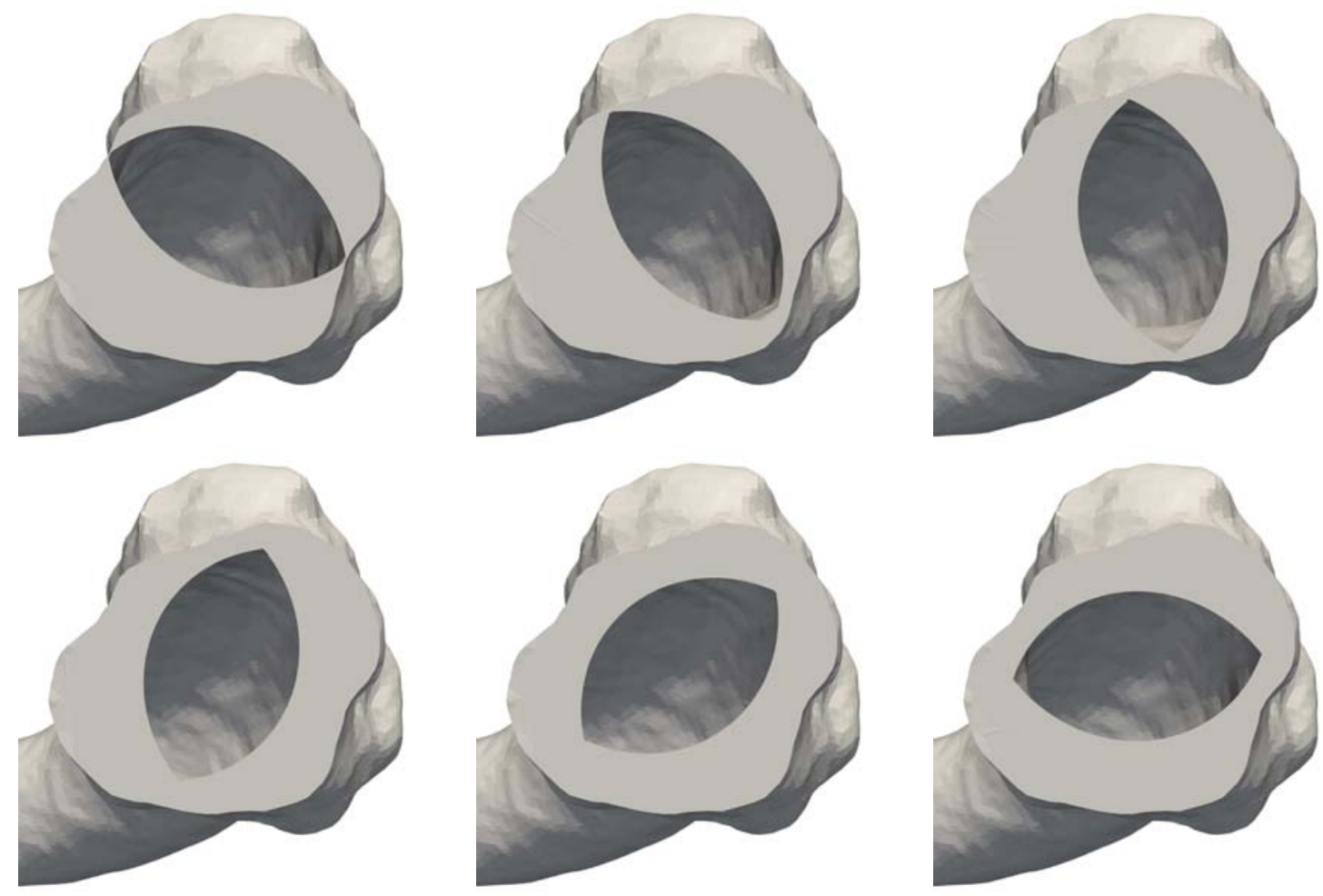

Figure 3: The six considered configurations for B2: B2-h12 (left, up), B2-h1 (center, up), B2-h2 (right, up), B2-h3 (left, bottom), B2-h4 (center, bottom) and B2-h5 (right, bottom).
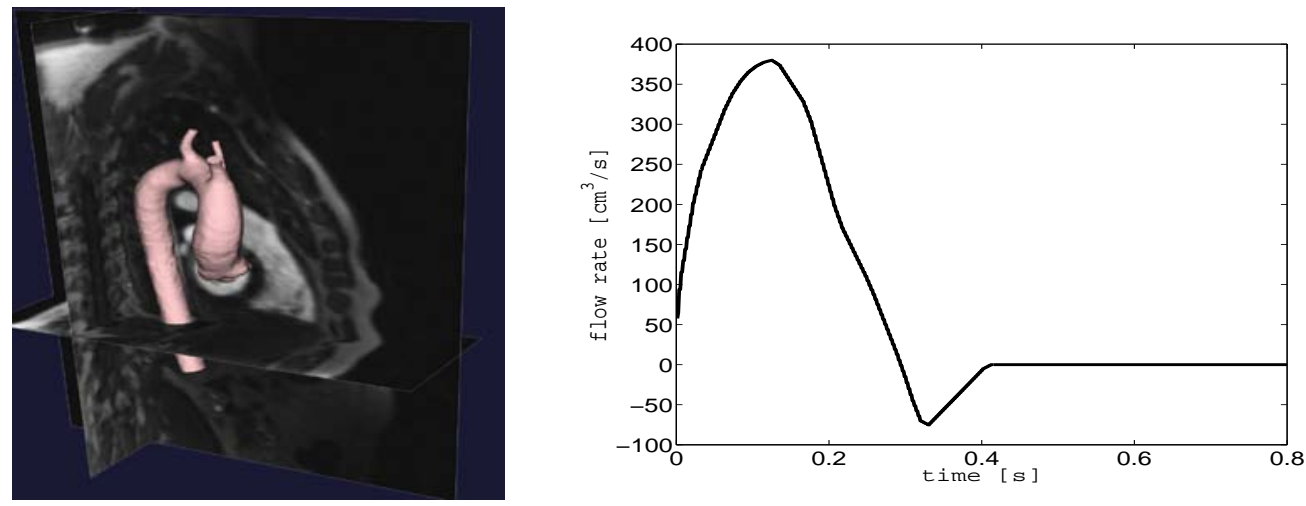

Figure 4: Left: example of the relationship between patient MRI and completed vascular modeling process. Right: flow rate boundary condition prescribed at the inlet of the ascending aorta as representative of the heart action (taken from [2]). 


\section{$2.2 \quad$ Numerical simulations}

Unsteady and laminar numerical simulations are performed in the computational domains defined in the previous section using the finite element code LifeV (a numerical analysis library jointly developed by MOX-Politecnico di Milano, INRIA-Paris, CMCS-EPFL-Lausanne, and more recently at the Emory University, see http://www.lifev.org). To speed up the analysis, we resort to a parallel execution on a 48-processor architecture.

In all simulations, blood was considered as Newtonian, homogeneous, and incompressible, so that the Navier-Stokes equations for incompressible fluids are used for the mathematical description (see, e.g., [15]). Blood viscosity is set to 0.035 Poise and the density equal to $1.0 \mathrm{~g} / \mathrm{cm}^{3}$. For time discretization, the time step was chosen equal to $0.01 \mathrm{~s}$. Being interested in hemodynamics at systolic ejection, the vessel wall is considered rigid and in a fixed configuration, with the geometry corresponding to the systolic ejection phase of the cardiac cycle. Valve leaflets dynamics is not modeled as assumed not influencing the direction of the systolic jet at peak systole. Valve opening and closing are therefore modeled in an on/off modality. In particular, the valve is completely open for the first $0.4 \mathrm{~s}$ of the heartbeat and closed for the remaining time. At the inlet, the physiological flow rate depicted in Figure 4, right, and taken from [2], is chosen as representative of the heart pumping action. To prescribe the flow rate waveform at the valve's opening in order to minimally influence the computed flow field, we employ a Lagrange multipliers' approach (see $[14,30]$ for further details). At the oulets, we prescribe a zero-stress (homogeneous Neumann) condition.

Finally, we want to highlight that, as pointed out in [21], blood flow disturbances are only present near the aortic valve, being absent in the ascending aorta. Thus, in view of our purposes, we assume laminar flow (see also $[17,20]$ ).

\subsection{Indices for the comparison of different configurations}

Since the main aim of this paper is the quantitative comparison of hemodynamics among different BAV configurations, in this section we introduce two new indices for a quantification of the WSS in the ascending aorta.

To this end, let $A$ be the area of the region of the wall of the ascending aorta, and let $S(t)$ be a threshold of the wall shear stress $(W s s(t))$ determined in this region (measured for example in Pascal), and where $t$ is time. Moreover, we introduce the quantity $A_{Q}(t, S)$, which is the area of the region of interest such that at time $t$ the WSS is greater than $S(t)$,

$$
A_{Q}(t, S):=\int_{A} \chi(t, S) d \boldsymbol{x}, \quad \chi(t, S)=\left\{\begin{array}{l}
1, \text { if } W s s(t) \geq S(t), \\
0, \text { if } W s s(t)<S(t),
\end{array}\right.
$$

for a given function of time $S(t)$. We introduce the following two functions:

$$
\begin{aligned}
Q_{1}(t, S) & :=\frac{A_{Q}(t, S)}{A}, \\
Q_{2}(t, S) & :=\frac{\int_{A_{Q}(t, S)} W s s(t) d \boldsymbol{x}}{A} .
\end{aligned}
$$

The quantity $Q_{1}(t, S)$ gives the percentage of the area of the region of interest such that at time $t$ WSS is greater than $S(t)$, whilst the quantity $Q_{2}(t, S)$ gives the mean of the WSS in the region where, at time $t$, it is greater than $S(t)$. 
Furthermore, we introduce the following global indices, namely the maximum of quantities $Q_{j}(t, S), j=$ 1,2 , over the cardiac beat

$$
Q_{j}^{\mathrm{MAx}}(S):=\max _{t \in[0, T]} Q_{j}(t, S), j=1,2,
$$

and the mean value of $Q_{j}(t, S), j=1,2$, over the cardiac beat

$$
Q_{j}^{\mathrm{MEAN}}(S):=\frac{1}{T} \int_{0}^{T} Q_{j}(t, S) d t, j=1,2 .
$$

Another specific behaviour of the blood flow downstream bicuspid valves is the asymmetry of the jet entering in the aorta with respect to the tricupid case, where the flow rate is more aligned with the vessel axis (see [31]). For this reason, in order to quantify this asymmetry with a summarizing index, we map the velocity magnitude field defined on a slice orthogonal to the vessel axis onto the circle of radius equal to 1 . On this circle, we then compute the distance $D$ between the point corresponding to the maximum velocity and the center of the circle (see Figure 5), yielding a number between 0 and 1 . Obviously, a high value of $D$ means that the asymmetry is pronounced, whilst values of $D$ approaching

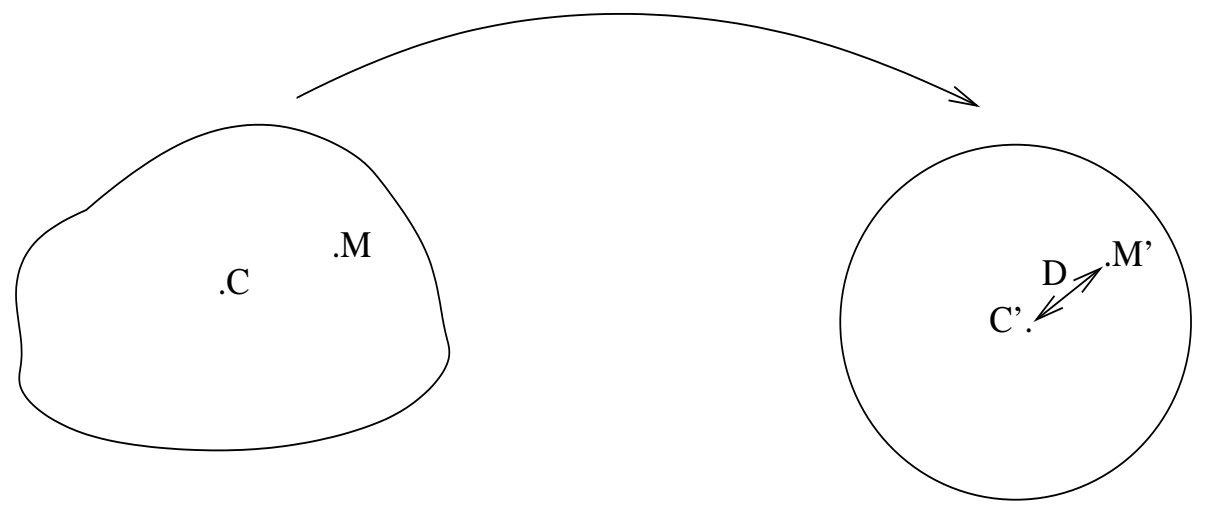

Figure 5: Mapping of a slice orthogonal to the aorta axis onto the circle of radius equal to 1 . The point $\mathrm{C}$ (the center) and the point $\mathrm{M}$ (where the maximum velocity occurs) are mapped onto C' and M', whose distance defines the index $D$.

zero are representative of more symmetric flows.

\section{Results}

In this Section we report the results obtained by performing Computational Fluid-Dynamics (CFD) numerical simulations for the different geometries as described in Section 2.2, and by then characterizing the resulting flow fields through the indices introduced in Section 2.3. In Section 3.1 we study the WSS distribution, aiming at finding relations between WSS intensity and location and different valve configurations. In Section 3.2 we study the degree of asymmetry of the blood flow entering the ascending aorta, again aiming at finding relationships with different valve configurations.

To get a first snapshot of the flow patterns developing within the ascending aorta, in Figure 6 we report the streamlines at systole $(t=0.12 s)$ for the tricuspid configuration and for the stenotic BAV con- 
figuration B3. We observe a higher degree of asymmetry and ricirculation zones in the latter configuration compared with tricuspid.
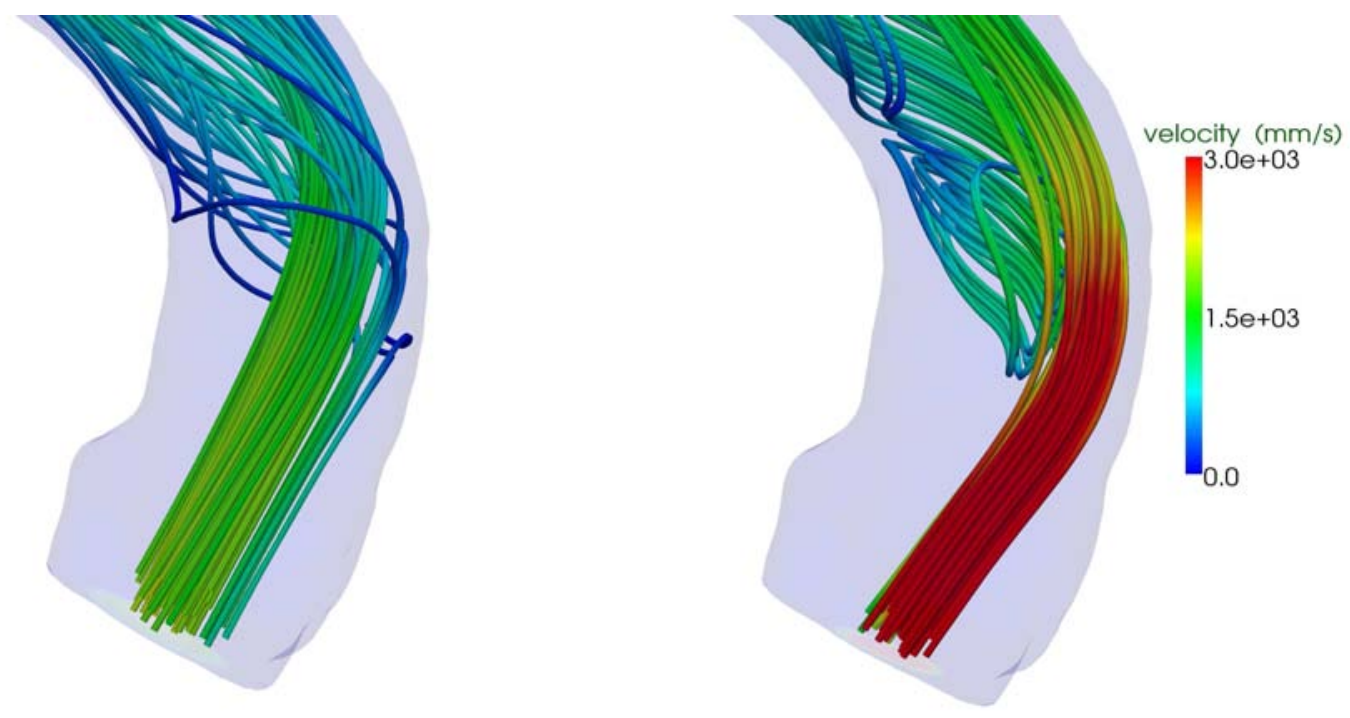

Figure 6: Streamlines for the tricuspid configuration (left) and for B3 (right) - $\mathrm{t}=0.12 \mathrm{~s}$.

\subsection{Wall shear stress analysis}

In Figure 7, we report the WSS at time $t=0.016 \mathrm{~s}$. The maximum WSS for the tricuspid case is of about $1.5 \mathrm{~Pa}$, which is in accordance with values already reported in the literature (see, e.g., [9, 17]). We observe a clear increase in WSS in the bicuspid valves with respect to the tricuspid case. Moreover, comparing $\mathrm{B} 1$ to $\mathrm{B} 2$ the increase is again evident, whilst from B2 to B3 it is less pronounced. Another clear pattern identified in these results is the different location of the region of higher WSS among the BAV configurations. In particular, for decreasing areas of the orifice, the region of higher WSS moves towards the aortic root.

In order to quantitatively characterize aortic hemodynamics according to the indices proposed in the previous section, we consider the region of interest shown in Figure 9, left. In Figure 10 we show the values of the quantities $Q_{1}(t, S)$ and $Q_{2}(t, S)$ for three constant values of the threshold, namely $S=1 P a, 2 P a, 3 P a$. In Table 1 we report the value of the four indices $Q_{i}^{\mathrm{MAX}}$ and $Q_{i}^{\mathrm{MEAN}}, i=1,2$, for these values of $S$. These results consistently show highest values of the indices for the bicuspid configurations compared to tricuspid. Moreover, among the bicuspid configurations, values of indices increase for decreasing areas. These results also show that the qualitative behavior of the indices is essentially independent of the choice of the threshold $S$. For this reason, in what follows we will consider a single value of $S$.

In order to characterize WSS patterns at different levels of the aorta, we have considered the following anatomical locations: annulus ( $A 1$ ), the Valsalva sinus $(A 2)$, the sino-tubolar junction (STJ, $A 3$ ) and the mid-ascending aorta $(A 4)$ (see Figure 9, right). In Table 2 we report the values of the four proposed 


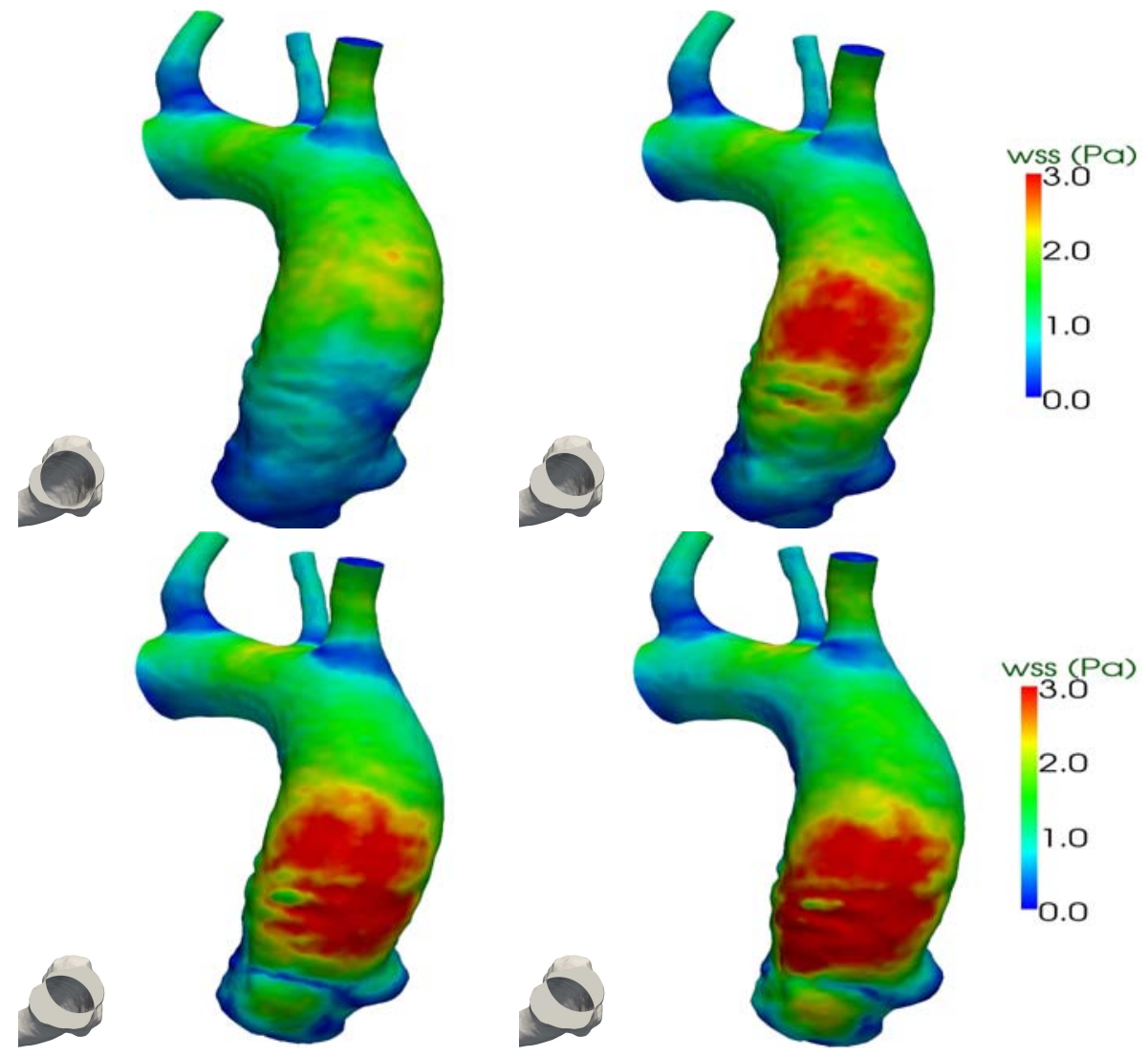

Figure 7: Wall shear stress (in Pascal) at $t=0.016 \mathrm{~s}$ : TRI configuration (left, up), B1 configuration (right, up), B2 configuration (left, bottom) and B3 configuration (right, bottom). 


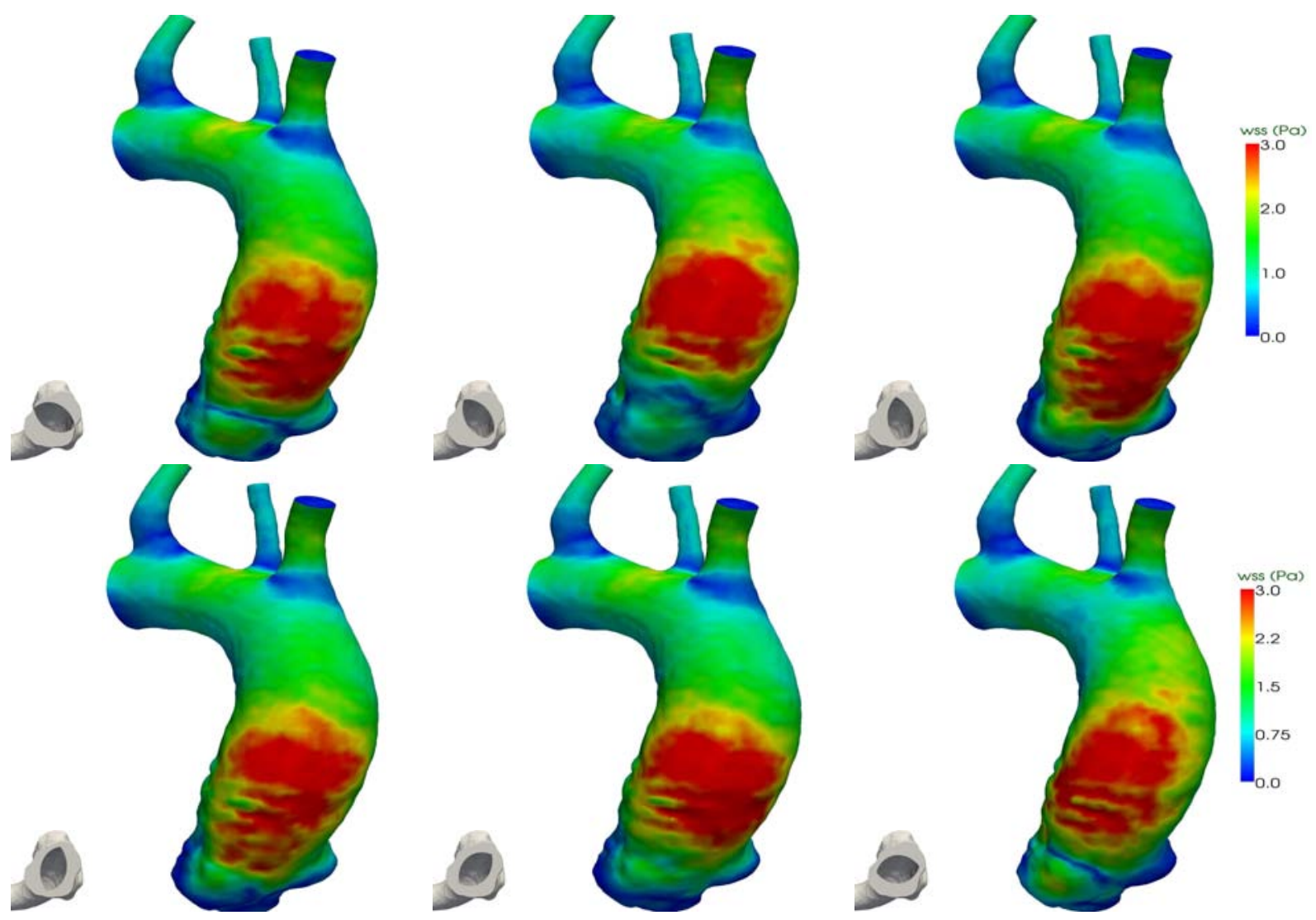

Figure 8: Wall shear stress (in Pascal) at $t=0.016 \mathrm{~s}$ : B2-h12 (left, up), B2-h1 (center, up), B2-h2 (right, up), B2-h3 (left, bottom), B2-h4 (center, bottom) and B2-h5 (right, bottom). 

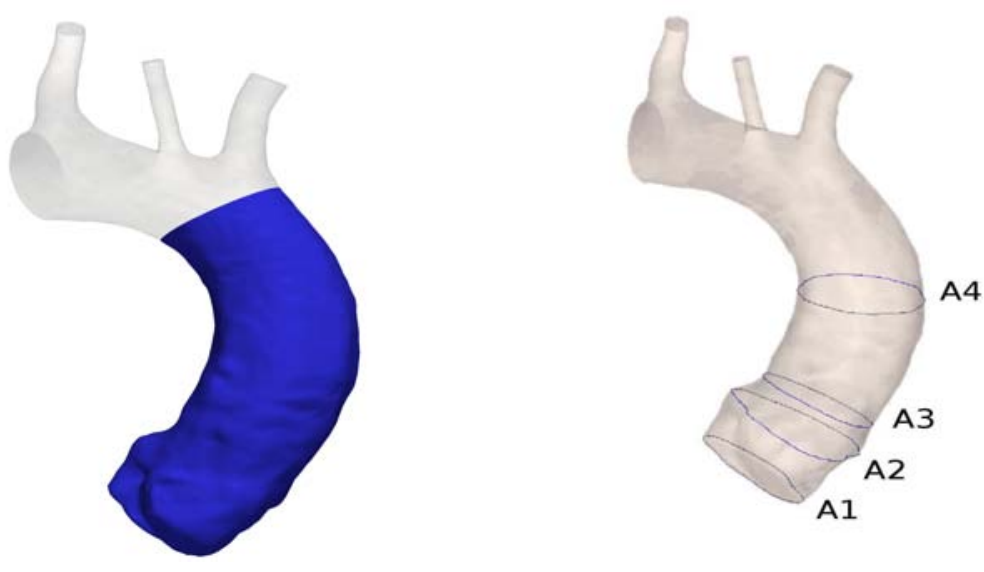

Figure 9: Left: region of interest where the quantities $Q_{j}$ are computed. Right: Four different locations where the fluid-dynamics quantities are computed. From the bottom to the up: annulus, Valsalva sinus, STJ, mid ascending aorta.
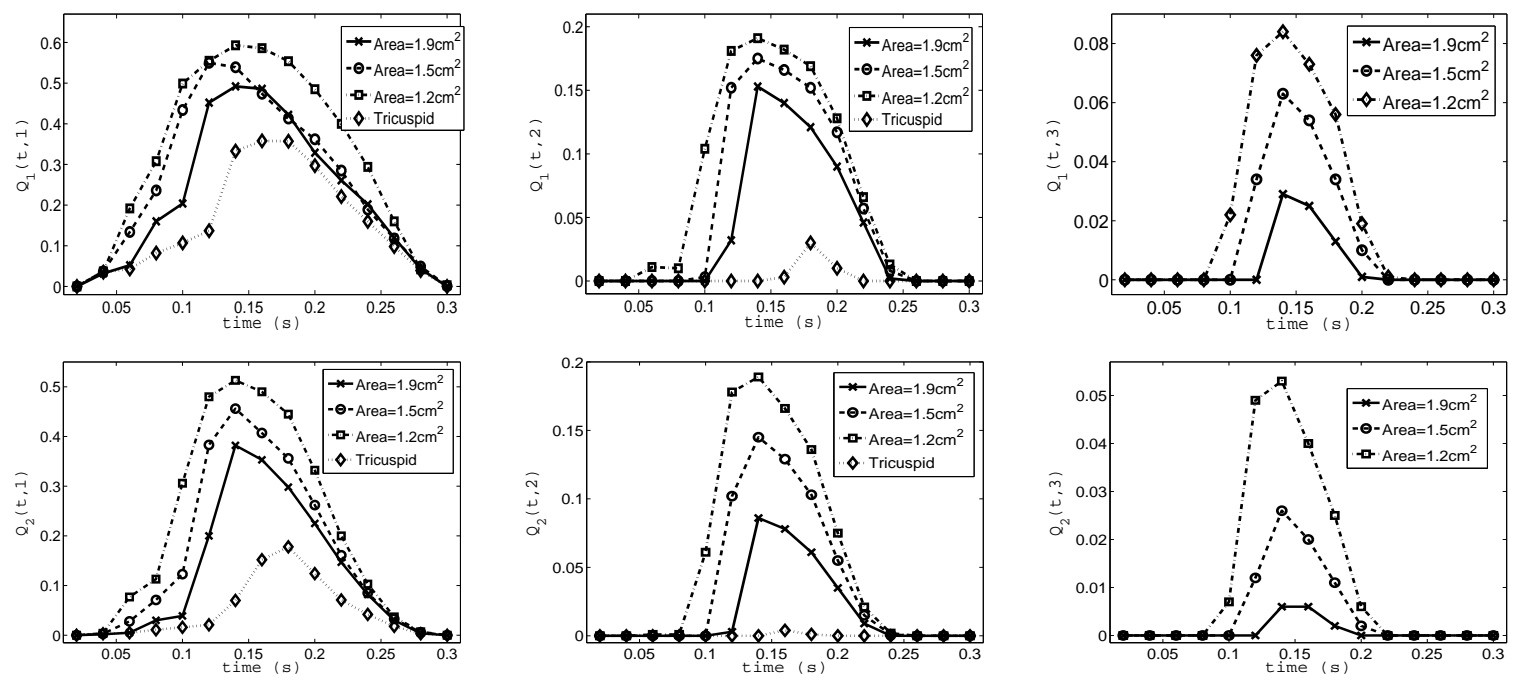

Figure 10: Quantities $Q_{1}(t, 1), Q_{1}(t, 2), Q_{1}(t, 3)$ (up) and $Q_{2}(t, 1), Q_{2}(t, 2), Q_{2}(t, 3)$ (bottom) for the four different configurations. 


\begin{tabular}{|c|c|c|c|c|}
\hline & TRI & B1 & B2 & B3 \\
\hline$Q_{1}^{\mathrm{MAX}}(1)$ & 0.358 & 0.492 & 0.550 & 0.593 \\
\hline$Q_{1}^{\mathrm{MAX}}(2)$ & 0.030 & 0.153 & 0.175 & 0.191 \\
\hline$Q_{1}^{\mathrm{MAX}}(3)$ & 0.0 & 0.029 & 0.063 & 0.084 \\
\hline$Q_{1}^{\mathrm{MEAN}}(1)$ & 0.162 & 0.230 & 0.270 & 0.336 \\
\hline$Q_{1}^{\mathrm{MEAN}}(2)$ & 0.003 & 0.042 & 0.059 & 0.075 \\
\hline$Q_{1}^{\mathrm{MEAN}}(3)$ & 0.0 & 0.005 & 0.014 & 0.024 \\
\hline$Q_{2}^{\mathrm{MAX}}(1)$ & 0.178 & 0.382 & 0.456 & 0.513 \\
\hline$Q_{2}^{\mathrm{MAX}}(2)$ & 0.004 & 0.086 & 0.145 & 0.189 \\
\hline$Q_{2}^{\mathrm{MAX}}(3)$ & 0.0 & 0.006 & 0.026 & 0.053 \\
\hline$Q_{2}^{\mathrm{MEAN}}(1)$ & 0.051 & 0.128 & 0.169 & 0.222 \\
\hline$Q_{2}^{\mathrm{MEAN}}(2)$ & 0.0 & 0.019 & 0.039 & 0.059 \\
\hline$Q_{2}^{\mathrm{MEAN}}(3)$ & 0.0 & 0.001 & 0.005 & 0.013 \\
\hline
\end{tabular}

Table 1: Values of the four proposed indices for $S=1,2,3$. 
indices for $S=1$, for the four configurations at the four different locations. In these results, all four indices show that the WSS at the annulus is higher for the tricuspid configuration, being close to zero for the bicuspid configurations. Viceversa, at the other three levels, WSS is lower for the tricuspid configuration. Also, among the bicuspid configurations, the values of the indices show that the WSS is higher for decreasing values of the area, with the exception of index $Q_{1}^{\operatorname{MAX}}(1)$ for levels A2 and A3 and of $Q_{2}^{\mathrm{MAX}}(1)$ for level A4.

In Figure 8 we report the WSS at time $t=0.16 s$ for the six rotated configurations of B2. These results show that there is no pronounced difference among the six cases, which in any case are characterized by exposure to high WSS compared to the tricuspid case.

Then, we have computed the four proposed indices for the six rotated configurations of B2, by setting $S=2 \mathrm{~Pa}$ as threshold. The results are reported in Table 3. These values do not seem to differ markedly among configurations, although the latero-lateral configurations B2-h2 and B2-h4 are associated to slightly higher values compared to the rest.

Finally, in Table 4 we show the values of the indices for the six rotated configurations, obtained at the four different levels, by setting $S=1 P a$ as threshold. These results show that all the values are small at the annulus, whilst the configurations B2-h3, B2-h4 and B2-h5 show the highest values at the Valsalva sinus for all the four indices. At the sino-tubular junction the highest values are associated to configurations B2-h2, B2-h3 and B2-h4. Finally, at the mid-ascending aorta $Q_{1}$ shows that the WSS is highest for the antero-posterior configurations, whilst the values of $Q_{2}$ shows an almost uniform value of the WSS. Last, we point out that in all configurations the four indices are highest at the STJ and mid-ascending aorta levels.

\subsection{Flow asymmetry analysis}

In Figure 11 we report the velocity field at time $t=0.12 \mathrm{~s}$ for the four configurations, at three of the different locations of the aorta, namely at the Valsalva sinus, STJ and mid-ascending aorta. On the right, we also report the respective velocity vectors for each configuration. In Table 5 we report the values of the index $D$ obtained for the four configurations at the valsalva sinus, STJ and Mid-ascending aorta. The results clearly show, for all the three levels considered, an increment of the asymmetry of the blood flow for decreasing values of the area. In particular, configurations B2 and B3 have more or less the same degree of asymmetry which is higher with respect to the one obtained in B1.

In Figure 12 we report the velocity field at time $t=0.12 \mathrm{~s}$ for the six configurations, at the three different locations of the aorta (Valsalva sinus, STJ and mid-ascending aorta), and the velocity vectors (on the right).

Finally in Table 6 we report the values of index $D$ for the six configurations at the levels A2, A3 and A4. These results show that the quantitative differences in the degree of asymmetry for the six orientations are not markedly pronounced, even though configuration B2-h1 seems to have a lower degree of asymmetry at levels A2 and A3.

\section{Discussion}

Numerical approaches for the study of haemodynamics in the ascending aorta have been employed successfully in the recent past. We cite for example [17,20,28], where an open tricuspid valve configuration is modeled. Other works investigated the mechanism of valve openining in the bicuspid configuration, as in $[7,34]$. These works were focused on the opening dynamics of the leaflets in realistic, although 


\begin{tabular}{|c|c|c|c|c|c|}
\hline & & TRI & B1 & B2 & B3 \\
\hline \multirow{4}{*}{ A1 } & $Q_{1}^{\operatorname{MAX}}(1)$ & 0.102 & 0.051 & 0.016 & 0.024 \\
\hline & $Q_{1}^{\mathrm{MEAN}}(1)$ & 0.051 & 0.026 & 0.003 & 0.005 \\
\hline & $Q_{2}^{\mathrm{MAX}}(1)$ & 0.025 & 0.019 & 0.001 & 0.001 \\
\hline & $Q_{2}^{\mathrm{MEAN}}(1)$ & 0.011 & 0.006 & 0.0 & 0.0 \\
\hline \multirow{4}{*}{ A2 } & $Q_{1}^{\mathrm{MAX}}(1)$ & 0.040 & 0.214 & 0.129 & 0.845 \\
\hline & $Q_{1}^{\mathrm{MEAN}}(1)$ & 0.008 & 0.016 & 0.041 & 0.167 \\
\hline & $Q_{2}^{\mathrm{MAX}}(1)$ & 0.002 & 0.023 & 0.046 & 0.461 \\
\hline & $Q_{2}^{\mathrm{MEAN}}(1)$ & 0.0 & 0.002 & 0.011 & 0.088 \\
\hline \multirow{4}{*}{ A3 } & $Q_{1}^{\mathrm{MAX}}(1)$ & 0.161 & 0.194 & 0.963 & 0.912 \\
\hline & $Q_{1}^{\mathrm{MEAN}}(1)$ & 0.079 & 0.035 & 0.173 & 0.362 \\
\hline & $Q_{2}^{\mathrm{MAX}}(1)$ & 0.046 & 0.019 & 0.524 & 0.559 \\
\hline & $Q_{2}^{\mathrm{MEAN}}(1)$ & 0.018 & 0.003 & 0.056 & 0.175 \\
\hline \multirow{4}{*}{$\mathrm{A} 4$} & $Q_{1}^{\mathrm{MAX}}(1)$ & 0.669 & 0.731 & 0.801 & 0.826 \\
\hline & $Q_{1}^{\mathrm{MEAN}}(1)$ & 0.290 & 0.380 & 0.443 & 0.465 \\
\hline & $Q_{2}^{\mathrm{MAX}}(1)$ & 0.322 & 0.994 & 0.953 & 0.869 \\
\hline & $Q_{2}^{\mathrm{MEAN}}(1)$ & 0.090 & 0.364 & 0.394 & 0.367 \\
\hline
\end{tabular}

Table 2: Values of the four proposed indices for $S=1$ at the four locations $A_{j}, j=1, \ldots, 4$. 

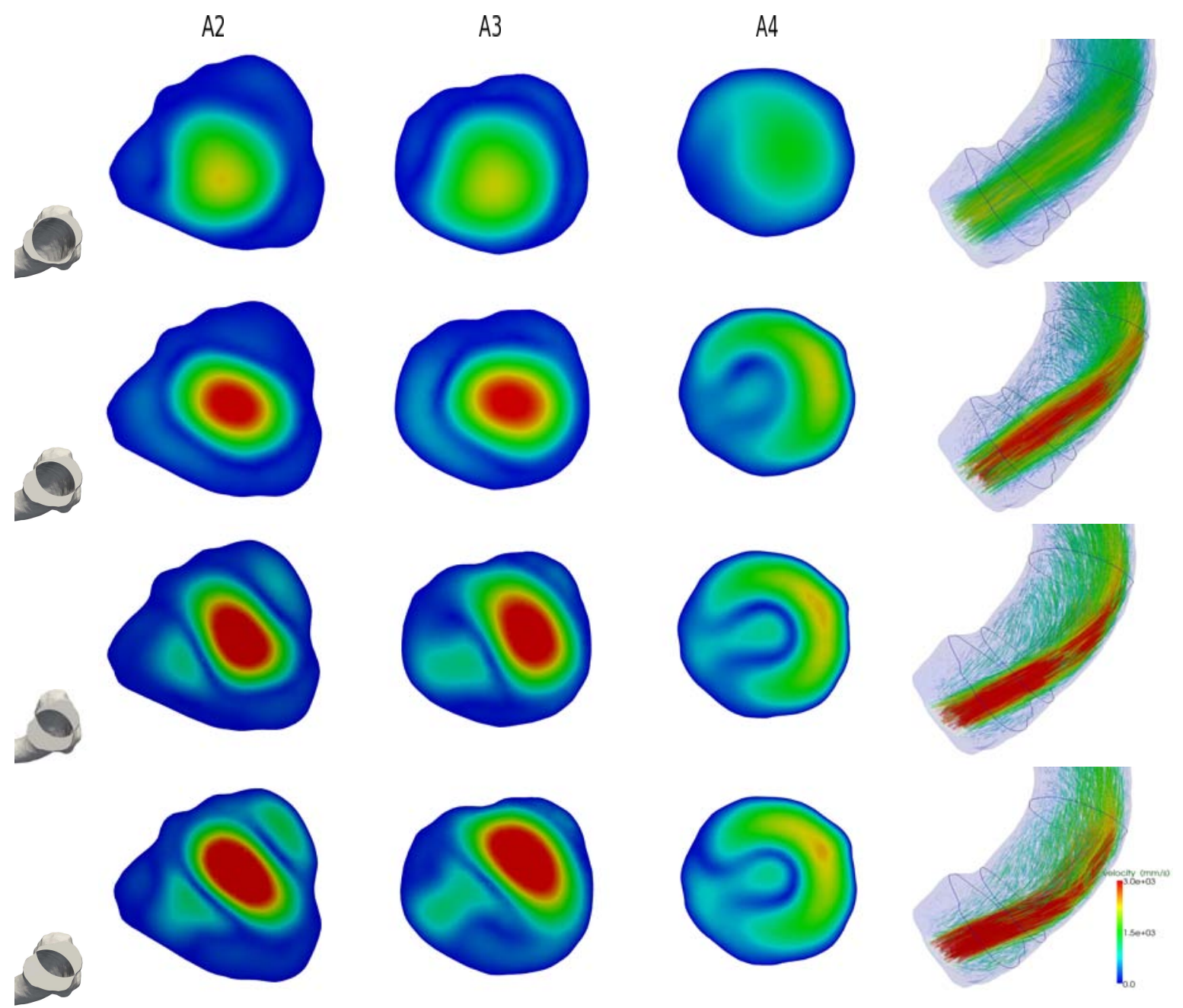

Figure 11: Velocity field at three different locations and velocity vectors in the zone of interest, for the four configurations. From up to bottom: TRI, B1, B2, B3. From left to right: valsalva sinus, STJ, mid ascending aorta, velocity vectors $-\mathrm{t}=0.12 \mathrm{~s}$. 


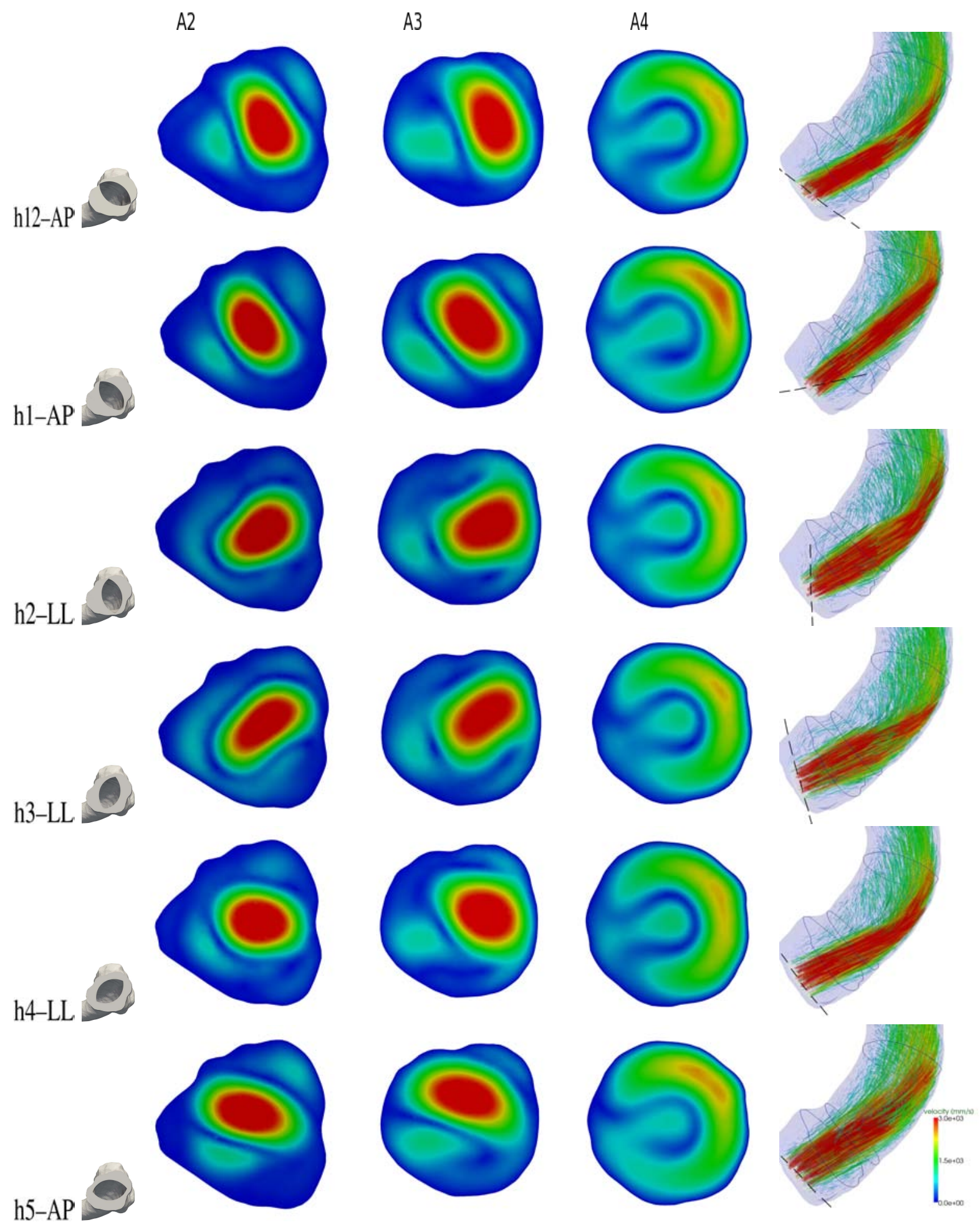

Figure 12: Velocity field at three different locations 15 and velocity vectors in the zone of interest, for the six configurations. From up to bottom: B2-h12, B2-h1, B2-h2, B2-h3, B2-h4, B2-h5. From left to right: Valsalva sinus, STJ, mid ascending aorta, orthogonal section - $\mathrm{t}=0.12 \mathrm{~s}$. 


\begin{tabular}{c|cccccc} 
& B2-h12 & B2-h1 & B2-h2 & B2-h3 & B2-h4 & B2-h5 \\
\hline \hline$Q_{1}^{\operatorname{MAX}}(2)$ & 0.175 & 0.161 & 0.192 & 0.171 & 0.186 & 0.170 \\
$Q_{1}^{\operatorname{MEANN}}(2)$ & 0.059 & 0.053 & 0.074 & 0.065 & 0.067 & 0.067 \\
$Q_{2}^{\operatorname{MAX}}(2)$ & 0.145 & 0.136 & 0.171 & 0.132 & 0.171 & 0.134 \\
$Q_{2}^{\operatorname{MEAN}}(2)$ & 0.039 & 0.036 & 0.053 & 0.040 & 0.050 & 0.034
\end{tabular}

Table 3: Values of the four proposed indices for $S=2$ for the six rotated configurations.

not patient-specific, geometries. The first work also includes a fluid-dynamics study, but only across the valve, without exploring the effects in the ascending aorta.

To the best of the authors' knowledge, in this paper we present the first application of computational methods to solve the clinical question of aortic aneurysms in BAV patients. We have previously (see [31]) used this methodology to study the differences in WSS exposure among normal tricuspid valve and two normally functioning BAV configurations. In that work, we pointed out that WSS exposure in BAV configurations was much higher than in the tricuspid model. This confirmed some findings $[3,5]$ that in these cases the risk of aneurysm formation might be high, and supported the hemodynamic hypothesis [23], which ascribes to the abnormal valve orifice shape (as found in BAV geometry) the generation of abnormal flow patterns through the ascending aorta and high WSS.

In this paper, bicuspid valve area and orientation were varied to look for potential relationships between BAV geometry and location and magnitude of WSS, asymmetry of the jet, and to propose indices for the quantification of WSS and asymmetry and for the potential prediction of the risk of aneurysm formation for the single BAV geometry. These indices are easily computable with finite element analysis. Moreover, the index related to the asymmetry could be computed, more routinely, also with phase contrast magnetic resonance imaging.

In this work, we decided to consider just one geometry and to set up all the studied configurations on it. This choice, besides simplicity reasons, was performed in order to investigate the sole effect of the valve configuration on the fluid-dynamics in isolation.

We first observe (Figure 10 and Table 1) that, for any choice of index and threshold, the smaller is the area of the valve, the higher is the value of the index and therefore the exposure at high WSS. This might be an obvious finding, as it is known from medical literature and clinical data that tricuspid patients with aortic stenosis are of risk of experiencing post-stenotic aortic aneurysms [4]. However, these results seem to be interestingly applicable also to the case of BAV. Moreover, we observe that even for non-stenotic bicuspid configurations (B1 and B2) WSS exposure is much higher than in tricuspid configuration, thus confirming the hypothesis that also in healthy bicuspid configurations the risk of aneurysm formation may be high. In this light, these results represent a preliminary though potentially important observation drawn from the proposed indices.

When we come to investigate the exposure to WSS at different aortic levels (Table 2) for B1, B2 and $\mathrm{B} 3$, we find that the higher WSS for all geometries $\left(Q_{2}^{\mathrm{MAX}}\right.$ and $\left.Q_{2}^{\mathrm{MEAN}}\right)$ are found at the ascending aorta portion (A4), irrespective of bicuspid orifice area. Interestingly, the latter is the aortic segment which is more prone to dilate and become aneurysmatic in BAV patients [25]. Regarding WSS distribution indices 


\begin{tabular}{|c|c|c|c|c|c|c|c|}
\hline & & B2-h12 & B2-h1 & B2-h2 & B2-h3 & B2-h4 & B2-h5 \\
\hline \multirow{4}{*}{ A1 } & $Q_{1}^{\mathrm{MAX}}(1)$ & 0.016 & 0.045 & 0.0 & 0.0 & 0.0 & 0.0 \\
\hline & $Q_{1}^{\mathrm{MEAN}}(1)$ & 0.003 & 0.026 & 0.0 & 0.0 & 0.0 & 0.0 \\
\hline & $Q_{2}^{\mathrm{MAX}}(1)$ & 0.001 & 0.044 & 0.0 & 0.0 & 0.0 & 0.0 \\
\hline & $Q_{2}^{\mathrm{MEAN}}(1)$ & 0.0 & 0.017 & 0.0 & 0.0 & 0.0 & 0.0 \\
\hline \multirow{4}{*}{$\mathrm{A} 2$} & $Q_{1}^{\mathrm{MAX}}(1)$ & 0.129 & 0.037 & 0.063 & 0.656 & 0.682 & 0.683 \\
\hline & $Q_{1}^{\mathrm{MEAN}}(1)$ & 0.041 & 0.007 & 0.011 & 0.088 & 0.070 & 0.111 \\
\hline & $Q_{2}^{\mathrm{MAX}}(1)$ & 0.046 & 0.003 & 0.004 & 0.206 & 0.236 & 0.189 \\
\hline & $Q_{2}^{\mathrm{MEAN}}(1)$ & 0.011 & 0.0 & 0.001 & 0.021 & 0.020 & 0.030 \\
\hline \multirow{4}{*}{ A3 } & $Q_{1}^{\mathrm{MAX}}(1)$ & 0.963 & 1.000 & 0.928 & 0.990 & 1.000 & 0.841 \\
\hline & $Q_{1}^{\mathrm{MEAN}}(1)$ & 0.173 & 0.140 & 0.283 & 0.347 & 0.257 & 0.247 \\
\hline & $Q_{2}^{\mathrm{MAX}}(1)$ & 0.524 & 0.644 & 0.641 & 0.663 & 0.699 & 0.608 \\
\hline & $Q_{2}^{\mathrm{MEAN}}(1)$ & 0.056 & 0.058 & 0.188 & 0.147 & 0.117 & 0.098 \\
\hline \multirow{4}{*}{$\mathrm{A} 4$} & $Q_{1}^{\mathrm{MAx}}(1)$ & 0.801 & 0.916 & 0.714 & 0.710 & 0.762 & 0.896 \\
\hline & $Q_{1}^{\mathrm{MEAN}}(1)$ & 0.443 & 0.444 & 0.412 & 0.415 & 0.436 & 0.466 \\
\hline & $Q_{2}^{\mathrm{MAX}}(1)$ & 0.953 & 1.296 & 1.013 & 0.960 & 1.007 & 1.057 \\
\hline & $Q_{2}^{\mathrm{MEAN}}(1)$ & 0.394 & 0.457 & 0.408 & 0.392 & 0.413 & 0.366 \\
\hline
\end{tabular}

Table 4: Values of the four proposed indices for $S=1$ at the four locations $A_{j}, j=1, \ldots, 4$, for the six rotated configurations. 


\begin{tabular}{c|ccc} 
& A2 & A3 & A4 \\
\hline \hline TRI & 0.32 & 0.26 & 0.20 \\
B1 & 0.09 & 0.19 & 0.61 \\
B2 & 0.21 & 0.33 & 0.69 \\
B3 & 0.22 & 0.35 & 0.70
\end{tabular}

Table 5: Values of the index $D$ at the three locations valsalva sinus (A2), STJ (A3) and Mid-ascending aorta (A4).

\begin{tabular}{c|ccc} 
& A2 & A3 & A4 \\
\hline \hline B2-h12 & 0.21 & 0.33 & 0.69 \\
B2-h1 & 0.10 & 0.09 & 0.67 \\
B2-h2 & 0.29 & 0.32 & 0.68 \\
B2-h3 & 0.19 & 0.28 & 0.68 \\
B2-h4 & 0.24 & 0.32 & 0.68 \\
B2-h5 & 0.12 & 0.23 & 0.68
\end{tabular}

Table 6: Values of the index $D$ at the three locations Valsalva sinus (A2), STJ (A3) and mid-ascending aorta (A4), for the six rotated configurations.

$\left(Q_{1}^{\mathrm{MAX}}\right.$ and $\left.Q_{1}^{\mathrm{MEAN}}\right)$, also shown in Table 2, we notice that for configurations B2 and B3 the values are high also for the STJ section (A3). This could be easily explained as this is the narrowest segment (apart from aortic annulus) after a sinus-like section (A2, Valsalva sinuses), in which a physiological diversion of the blood jet is experienced, resulting in an increase in WSS area indices even for the tricuspid configuration. Here, values related to tricuspid and B1 configurations are very different from B2 and B3, suggesting that in sino-tubular junction segment WSS values might be more dependent on valve area (larger area, less WSS) rather than on valve geometry (BAV vs. TAV). The latter consideration might be true also for the Valsalva sinus level (A2), with the only exception for the value of $Q_{1}^{\mathrm{MAX}}$ in B1. At the aortic annulus we notice that the indices are higher for increasing area of the valve, highest values being associated to the tricuspid model. The smaller the area, the stronger and more penetrating is the blood jet, and then viscous forces are relatively weaker at this level of aorta for the configurations with smaller area.

For what concerns bulk fluid-dynamics, from Figure 11 it is clear that the blood flow downstream the ascending aorta uniformly fills the ascending aorta for the tricuspid case, whilst it is confined in a small region, giving rise to a jet, in the bicuspid case. Moreover, by decreasing the area of the bicuspid valve, the jet is consistently more concentrated, which is in line with what expected from basic fluid dynamics. More interesting are the results on the asymmetry indices. In particular, Table 5 shows that the smallest the area of BAV, the highest is the value of $D$, with a maximum at the ascending aorta level, where the WSS values are larger, in agreement with findings shown in Table 2. Moreover, the tricuspid configuration at levels A2 and A3 highlights an asymmetry towards the ascending aortic wall concavity, that is the part of the wall internal with respect to the curvature of the ascending aorta. Viceversa, for bicuspid configurations the jet is not aligned with the axial direction, but it quickly shifts towards the ascending aortic wall convexity (the portion of the wall external with respect to the curvature, see also 
Figure 11), while increasingly larger vortices appear on the aortic concavity. By decreasing the area of the bicuspid orifice, the zone which the jet points to moves towards the aortic root. This fact is in accordance with the WSS distribution found in Figure 7, whose zone of higher intensity was confined in a region that moves towards the root by decreasing the area of the orifice. We also observe that configuration B1 features smaller values of $D$ in comparison with $B 2$ and $B 3$ at all the three levels $A 2, A 3$ and $A 4$.

While there are not many differences among configurations in Table 3, where the four proposed indices are shown with threshold $S=2 P a$ for the six rotated configurations of B2 (although latero-lateral geometries show slightly higher values), the results in Table 4 are more noteworthy. Latero-lateral configurations (mostly B2-h3 and B2-h4) show higher values of $Q_{1}$ at the Valsalva and sinotubular junction levels (A2 and A3), confirming the clinical finding that LL BAVs are prone to altered aortic proportions among these locations. Also, the asymmetry index $D$ is higher for the LL configurations (Table 6). The impression is that LL configurations are associated with higher velocity flow fields asymmetry and WSS, mainly at levels A2 and A3. This could explain the well described higher prevalence of tubular aneurysms (i.e. the ones where there is a uniform dilation of sinus of Valsalva, STJ and mid-ascending aorta) in these configurations. On the contrary, divergent aneuryms (i.e. those where maximum dilatation is at the STJ and/or at mid-ascending aorta) are more commonly found in patients with AP BAV morphology (see $[8,19])$. Accordingly, configurations B1-h1 and B1-h5, which in this model represent AP BAV orientation, show higher $Q_{1}$ (see Table 4) at the ascending aorta level, whilst the value of $Q_{2}$ shows an almost constant level of WSS.

By looking at these results, we point out that index $D$ could be an effective quantity for use in clinical settings, and a valid surrogate of impaired WSS exposure. Indeed, its computation is easy and, in principle, it could be obtained without performing any numerical simulation, but just by evaluating the anatomy and drawing some information about the velocity field, as feasible with clinically available phase-contrast MRI or cardiac Ultrasound technology.

\section{Conclusions, limitations and future perspectives}

The results of this work highlight that in normally functioning BAV the jet exiting from the orifice is characterized by a higher degree of asymmetry when the area decreases. This induces high WSS distribution in the ascending aortic wall convexity, and the zone of maximum WSS moves towards the root for decreasing area, in accordance with the degree of asymmetry. For the configuration B2, maximum WSS, flow velocity and asymmetry occur at mid-ascending aorta, regardless of the leaflet orientation. We observed high WSS distributions for LL configurations at the Valsalva sinus and STJ levels, in accordance with the clinical observation of prevalence of tubular aneurysms in these configurations. As the proposed indices are validated, they may prove helpful for a better planning of follow-up and surgical indications in BAV patients.

The first limitation of the present work concerns the dataset. We have considered only one geometry to build all valve configurations. This allowed to compare fluid-dynamics in those configurations, but a study on a larger dataset is of course mandatory.

The second limitation concerns the simplifying hypotheses introduced for the numerical simulations. In particular, the assumption of rigid walls is probably simplistic, since the motion of the aorta (due both to the interaction with blood and to the rigid movement of the heart) is not negligible. However, in [17] the authors highlighted that the differences in the WSS between the rigid and the compliant case could be up to $5-10 \%$. Moreover, we did not take into account the valve opening and closing mechanisms, and all numerical simulations were performed with an open valve. For both aspects, we do not expect 
that the moving walls or valve dynamics would have a major influence on the dynamics of the jet at the instant of peak ejection. In any case, a study which taking into account fluid-structure interaction and valve dynamics is mandatory as a future development.

In addition, the role of ascending aortic geometry (concavity and convexity/curvature) in WSS distribution and jet orientation, since BAV patients with short ascending aorta might be more prone to aneurysm formation, as well that of other factors such as heart frequency, stroke volume, (unhealthy) regurgitation at the valve's level and the asymmetry of the valve will deserve further investigation, in the search for potential players in the definition of a patient-specific predictor of aortic aneurysm formation in patients with BAV.

\section{Acknowledgments}

The authors would like to thank S. Merelli for her contribution in the generation of the configurations, and dr. G. Puppini for the medical images. The first author has been partially supported by the ERC Advanced Grant N.227058 MATHCARD.

\section{References}

[1] L. Antiga, M. Piccinelli, L. Botti, B. Ene-Iordache, A. Remuzzi, and D.A.Steinman. An image-based modeling framework for patient-specific computational hemodynamics. Med Biol Eng, 46:1097-1112, 2008 .

[2] A.P. Avolio. Multi-branched model of the human arterial system. Med Biol Eng Comp, 18:709-718, 1980.

[3] M. Bauer, H. Siniawski, M. Pasic, B. Schaumann, and R. Hetzer. Different hemodynamic stress of the ascending aorta wall in patients with bicuspid and tricuspid aortic valve. J Card Surg, 21(3):218-220, 2006.

[4] R.O. Bonow, B. Carabello, A.C. de Leon Jr, L.H. Edmunds Jr, B.J. Fedderly, M.D. Freed, W.H. Gaasch, C.R. McKay, R.A. Nishimura, P.T. O'Gara, R.A. O'Rourke, S.H. Rahimtoola, J.L. Ritchie, M.D. Cheitlin, K.A. Eagle, T.J. Gardner, A. Garson Jr, R.J. Gibbons, R.O. Russell, T.J. Ryan, and S.C. Smith Jr. Guidelines for the management of patients with valvular heart disease: executive summary: A report of the american college of cardiology/american heart association task force on practice guidelines (committee on management of patients with valvular heart disease). Circulation, 98:1949-1984, 1998.

[5] M. Cecconi, M. Manfrin, A. Moraca, R. Zanoli, P.L. Colonna, M.G. Bettuzzi, S. Moretti, and D. Gabrielli G.P. Perna. Aortic dimensions in patients with bicuspid aortic valve without significant valve dysfunction. Am J Cardiol, 95(2):292-294, 2005.

[6] M.J. Collins, V. Dev, and B.H. Strauss. Variation in the histopathological features of patients with ascending aortic aneurysms: a study of 111 surgically excised cases. J Clin Pathol, 61:519-523, 2008.

[7] C.A. Conti, A. Della Corte, E. Votta, L. Del Viscovo, C. Bancone, L.S. De Santo, and A. Redaelli. Biomechanical implications of the congenital bicuspid aortic valve: A finite element study of aortic 
root function from in vivo data. The Journal of Thoracic and Cardiovascular Surgery, 140:890-896, 2010.

[8] A. Della Corte, G. Romano, F. Tizzano, C. Amarelli, L.S. De Santo, M. De Feo, M. Scardone, G. Dialetto, F.E. Covino, and M. Cotrufo. Echocardiographic anatomy of ascending aorta dilatation: correlations with aortic valve morphology and function. Int J Cardiol, 113(3):320-326, 2006.

[9] E. P. Efstathopoulos, G. Patatoukasa, I. Pantos, O. Benekos, D. Katritsis, and N. L. Kelekis. Wall shear stress calculation in ascending aorta using phase contrast magnetic resonance imaging. investigating effective ways to calculate it in clinical practice. Physica Medica, 24(4):175-181, 2008.

[10] P.W. Fedak, T. David, M. Borger, S. Verma, and J. Butany R.D. Weisel. Bicuspid aortic valve disease: recent insights in pathophysiology and treatment. Expert Rev Cardiovasc Ther, 3(2):295$308,2003$.

[11] P.W. Fedak, M.P. de Sa, S. Verma, N. Nili, M.S. Kazemian, J. Butany, B.H. Strauss, R.D. Weisel, and T. David. Vascular matrix remodeling in patients with bicuspid aortic valve malformations: implications for aortic dilatation. J Thor Cardiovasc Surg, 126(3):797-805, 2003.

[12] P.W. Fedak, S. Verma, T.E. David, R.L. Leask R.D. Weisel, and J. Butany. Clinical and pathophysiological implications of a bicuspid aortic valve. Circulation, 106(8):900-904, 2002.

[13] B. Fernndez, A.C. Durn, T. Fernndez-Gallego M.C. Fernndez, M. Such M, and J.M. Arqu V. SansComa. Bicuspid aortic valves with different spatial orientations of the leaflets are distinct etiological entities. J Am Coll Cardiol, 54(24):2312-2318, 2009.

[14] L. Formaggia, J.-F. Gerbeau, F. Nobile, and A. Quarteroni. Numerical treatment of defective boundary conditions for the Navier-Stokes equation. SIAM Journal on Numerical Analysis, 40(1):376-401, 2002 .

[15] L. Formaggia, A. Quarteroni, and A. Veneziani (Eds.). Cardiovascular Mathematics - Modeling and simulation of the circulatory system. Springer, 2009.

[16] M.D. Hope, A.K. Meadows, T.A. Hope, K.G. Ordovas, G.P. Reddy, M.T. Alley, and C.B. Higgins. Images in cardiovascular medicine. evaluation of bicuspid aortic valve and aortic coarctation with 4d flow magnetic resonance imaging. Circulation, 117:2818-2819, 2008.

[17] S. Jin, J. Oshinski, and D.P Giddens. Effects of wall motion and compliance on flow patterns in the ascending aorta. Journal of Biomechanical Engineering, 125(3):347-354, 2003.

[18] T.C. Lee, Y.D. Zhao, D.W. Courtman, and D.J. Stewart. Abnormal aortic valve development in mice lacking endothelial nitric oxide sinthase. Circulation, 101:2345-2348, 2000.

[19] G.B. Luciani, A. Favaro, G. Casali, F. Santini, and A. Mazzucco. Reoperations for aortic aneurysm after the ross procedure. J Heart Valve Dis, 14(6):766-772, 2005.

[20] L. Morris, P. Delassus, A. Callanan, M. Walsh, F. Wallis, P. Grace, and T. McGloughlin. 3-d numerical simulation of blood flow through models of the human aorta. Journal of Biomechanical Engineering, 127(5):767-775, 2005. 
[21] W.W. Nichols and M.F. O'Rourke. McDonald's blood flow in arteries: Theoretical, experimental and clinical principles. Edward Arnold, 1998.

[22] S. Nistri, M.D. Sorbo, M. Marin, M. Palisi R. Scognamiglio, and G. Thiene. Aortic root dilatation in young men with normally functioning bicuspid aortic valves. Heart, 82(1):19-22, 1999.

[23] P.M. Den Reijer, D. Sallee III, P. van der Velden, E.R. Zaaijer, W.J. Parks, S. Ramamurthy, T.Q. Robbie, G. Donati, C. Lamphier, R. Beekman, and M.E. Brummer. Hemodynamic predictors of aortic dilatation in bicuspid aortic valve by velocity-encoded cardiovascular magnetic resonance. $J$ Cardiovasc Magn Res, 12:4, 2010.

[24] W.C. Roberts. The congenital bicuspid aortic valve. Am J Cardiol, 26:72-73, 1970.

[25] C.F. Russo, A. Cannata, M. Lanfranconi, E. Vitali, A. Garatti, and E. Bonacina. Is aortic wall degeneration related to bicuspid aortic valve anatomy in patients with valvular disease? $J$ Thorac Cardiovasc Surg, 136(4):937-942, 2008.

[26] H.Y. Sabet, W.D. Edwards, H.D. Tazelaar, and R.C. Daly. Congenitally bicuspid aortic valves: a surgical pathology study of 542 cases (1991 through 1996) and a literature review of 2,715 additional cases. Mayo Clin Proc, 74(1):14-26, 1999.

[27] B.M. Schaefer, M.B. Lewin, K.K. Stout, P.H. Byers, and C.M. Otto. Usefulness of bicuspid aortic valve phenotype to predict elastic properties of the ascending aorta. Am J Cardiol, 99(5):686-690, 2007.

[28] N. Shahcheraghi, H.A. Dwyer, A.Y. Cheer, A.I. Barakat, and T. Rutaganira. Unsteady and threedimensional simulation of blood flow in the human aortic arch. J Biomech Eng, 124(4):378-387, 2002 .

[29] H.H. Sievers and C. Schmidtke. A classification system for the bicuspid aortic valve from 304 surgical specimens. Journal of Thoracic and Cardiovascular Surgery, 133(5):1226-1233, 2007.

[30] A. Veneziani and C. Vergara. Flow rate defective boundary conditions in haemodinamics simulations. International Journal for Numerical Methods in Fluids, 47:803-816, 2005.

[31] F. Viscardi, C. Vergara, L. Antiga, S. Merelli, G. Puppini, A. Veneziani, G. Faggian, A. Mazzucco, and G.B. Luciani. Comparative finite-element model analysis of ascending aortic flow in bicuspid and tricuspid aortic valve. Artificial Organs, in press.

[32] C. Ward. Clinical significance of the bicuspid aortic valve. Heart, 83(1):81-85, 2000.

[33] E. Weigang, F.A. Kari, F. Beyersdorf, M. Luehr, C.D. Etz, A. Frydrychowicz, A. Harloff, and M. Markl. Flow sensitive four dimensional magnetic resonance imaging: flow patterns in ascending aortic aneurysms. Eur J Cardiothorac Surg, 34:11-16, 2008.

[34] E.J. Weinberg and M.R. Kaazempur Mofrad. A multiscale computational comparison of the bicuspid and tricuspid aortic valves in relation to calcific aortic stenosis. Journal of Biomechanics, 41(16):3482-3487, 2008. 